\title{
Mechanical Properties of Titanium-Aluminium Base Nanomultilayer Coatings
}

Totka Bakalova ${ }^{1}$, Nikolay Petkov $^{2}$, Tetiana Cholakova ${ }^{2}$, František Kaván ${ }^{1}$, Hristo Bahchedzhiev ${ }^{2}$

${ }^{1}$ Institute for Nanomaterials, Advanced Technologies and Innovation, Technical University of Liberec, Studentská 2, 461 17 Liberec, Czech Republic, E-mail: totka.bakalova@tul.cz, frantisek@kavan.eu

${ }^{2}$ Central Laboratory of Applied Physics, Bulgarian Academy of Sciences, 61, St. Peterburg Blvd. 4000 Plovdiv, Bulgaria. E-mail: petkovnik@gmail.com, ipfban-dve@mbox.digsys.bg, hristo_bah@abv.bg

Titanium-aluminium base nanomultilayer (NML) coatings are deposited by cathodic arc evaporation using pure titanium and aluminium with eighteen percent silicon cathodes. Each multilayer Ti-Al-Si-N structure consists of 49 bi-layers with different thickness. The external deposited layer is with five times longer growing period. The coatings are deposited at the pressure of $2 \mathrm{~Pa}$ and the substrate temperature of $400{ }^{\circ} \mathrm{C}$. Polished steel discs $(\varnothing 20$ $\mathrm{mm} \times \mathbf{5} \mathrm{mm}$ thick) are used as the substrate material.

This article presents an investigation of the surface morphology and mechanical properties of the coatings, particularly the adhesion and nanohardness. The AFM analysis indicates that the coatings are dense, with an average surface roughness in the range of $33 \div 58 \mathrm{~nm}$. The coating with the smaller value of average surface roughness exhibited a maximum hardness of $43 \mathrm{GPa}$. High calculated value of plasticity index $(H / E)$ is 0.104 . The scratch test results revealed that all the investigated coatings have very good adhesion in the normal loading interval from $1 \mathrm{~N}$ to $40 \mathrm{~N}$.

Keywords: cathodic arc evaporation, Ti-Al-Si-N, surface morphology, multilayer, hardness, adhesion.

\section{Acknowledgement}

The paper was supported in part by the project LO1201 through the financial support of the Ministry of Education, Youth and Sports in the framework of the targeted support of the "National Programme for Sustainability I" and the $O P R \& D I$ project “Centre for Nanomaterials, Advanced Technologies and Innovation" registration number CZ.1.05/2.1.00/01.0005.

\section{References}

[1] BARSHILIA, H. C., DEEPTHI, B., RAJAN, K. S. (2006). Vacuum, Vol. 81, pp. 479 - 488.

[2] BUDZYNSKI, P., SIELANKO, J., SUROWIEC, Z. (2008). Intermetallics, Vol. 16, pp. 987 - 994.

[3] SURESHA, S. J., BHIDE, R., JAYARAM, V., BISWAS, S. K. (2006). Materials Science and Engineering A, Vol. 429, pp. $252-260$.

[4] TENTARDINI, E. K., KWIETNIEWSKI, C., PERINI, F., BLANDO, E., HÜBLER, R., BAUMVOL, I. J. R. (2009). Surface \& Coatings Technology, Vol. 203, pp. $1176-1181$.

[5] JIANG, N., SHEN, Y. G., ZHANG, H. J., BAO, S. N., HOU, X. Y. (2006). Materials Science and Engineering B, Vol. 135, pp. $1-9$.

[6] CHEN, L., DU, Y., WANG, She. Q., WANG, Ai. J., XU, H. (2009). Materials Science and Engineering A, Vol. 502, pp. $139-143$.

[7] SINGH, K., LIMAYE, P. K., SONI, N. L., GROVER, A. K., AGRAWAL, R. G., SURI, A. K. (2005). Waer, Vol. 258, pp. $1813-1824$.

[8] MARQUES, L., CARVAlhO, S., VAZ, F., RAMOS, M. M. D., REBOUTA, L. (2009). Vacuum, Vol. 83, pp. $1240-1243$.

[9] LI, Y., SHIMADA, S., KIYONO, H., HIROSE, A. (2006). Acta Materialia, Vol. 54, pp. 2041 - 2048.

[10] MEI, F., SHAO, N., WEI, L., LI, G. (2005). Materials Letters, Vol. 59, pp. 2210 - 2213.

[11] CHEN, L., DU, Y., WANG, S. Q., J. Li. (2007). Int. Journal of Refractory Metals \& Hard Materials, Vol. 25, pp. $400-404$.

[12] CARVAlHO, S., REBOUtA, L., RIBEIRO, E., VAZ, F., TAVARES, C. J., ALVES, E., BARRADAS, N. P., RIVIERE, J. P. (2009). Vacuum, Vol. 83, pp. 1206 - 1212.

[13] TUILIER, M.-H., PAC, M.-J., COVAREL, G., ROUSSELOT, C., KHOUCHAF, L. (2007). Surface \& Coating Technology, Vol. 201, pp. $4536-4541$.

[14] CHEN, L., DU, Y., WANG, Ai J., WANG, S. Q., ZHOU, S. Z. (2009). Int. Journal of Refractory Metals \& Hard Materials, Vol. 27, pp. $718-721$. 
[15] OliVEIRA, J. C., MANAIA, A., CAVAlEIRO, A., VIEIRA, M. T. (2006). Surface \& Coating Technology, Vol. 201, pp. $4073-4077$.

[16] ZHAO, W., MEI, F., DONG, Y., LI, G. (2006). Journal of Materials Processing Tachnology, Vol. 176, pp. 179 182.

[17] OliVEIRA, J. C., MANAIA, A., CAVALEIRO, A. (2008). Thin Solid Films, Vol. 516, pp. 5032 - 5028.

[18] OliveIRA, J. C., MANAIA, A., DIAS, J. P., CAVAlEIRO, A., TEER, D., TAYLOR, S. (2006). Surface \& Coating Technology, Vol. 200, pp. 6583 - 6587.

[19] FERNANDES, C., CARVALHO, S., REBOUTA, L., VAZ, F., DENANNOT, M. F., PACAUD, J., RIVIERE, J. P., CAVALEIRO, A., (2008). Vacuum, Vol. 82, pp. 1470 - 1474.

[20] APERADOR, W., CAICEDO, J., ESPANA, C., CABRERA, G., AMAYA, C. (2010). Journal of Physics and Chemistry of Solids, Vol. 71, pp. $1754-1759$.

[21] PETKOV, N., BAHCHEDZHIEV, Hr., CHOLAKOVA, T. (2015). Eur. Phys. J. Appl. Phys., Vol. $70,30801$.

[22] ]NOVAK, M. (2012). Surface with High Precision of Roughness after Grinding. In: Manufacturing Technology, Vol. 12, No. 12, pp. 66-70, ISSN 1213-2489.

[23] SVOBODOVÁ, J., KRAUS, P. (2015). Influence of Chemical Pre-treatments Nanotechnology Based Applied to the Al Sheet on the Roughness and Morphology of the Surface. In: Manufacturing Technology, Vol. 15, No. 4, pp. $714-720$, ISSN 1213-2489.

[24] CHEN, L., DU, Y., YIN, F., LI, J. (2007). Int. Journal of Refractory Metals \& Hard Materials, Vol. 25, pp. 72 76.

[25] CHEN, L., DU, Y., MAYRHOFER, P. H., WANG, Sh. Q., LI, J. (2008). Surface \& Coating Technology, Vol. 202, pp. $5158-5161$.

[26] LEYLAND, A., MATTHEWS, A. (2000). Wear, Vol. 246, pp. $1-11$.

[27] WANG, Sh., CHEN, L., YANG, B., CHANG, K., DU, Y., LI, J., GANG, T. (2010). Int. Journal of Refractory Metals and Hard Materials, Vol. 28, pp. 593 - 596.

[28] CARVALHO, P. (2008). Development of new decorative coatings based on zirconium oxynitrides, Ph.D. Thesis, University of Minho, Portugal, http://hdl.handle.net/1822/8949. 